\title{
Microbiological and Biochemical Analysis of Methicillin-resistant Staphylococcus aureus Isolated from Patients Admitted in RIMS, Ranchi
}

\author{
Manju Boipai ${ }^{1}$ Krishna Chander Birua2*, N. P. Sahu³, L. B. Pandey ${ }^{3}$, Rameswari Beck ${ }^{4}$ Shashi Dinkar ${ }^{5}$
}

\begin{abstract}
Background: Since methicillin-resistant Staphylococcus aureus strains are resistant to multiple antibiotics, there is a possibility of extensive outbreaks which may be difficult to control. Early detection of methicillin-resistant Staphylococcus is important from patients and hospitals point of view. Materials and Methods: The present study was carried out in the Department of Microbiology, Rajendra Institute of Medical Sciences, Ranchi, clinical isolates of methicillin-resistant S. aureus strains were obtained from admitted patients of Rajendra Institute of Medical Sciences, Ranchi. The sources of isolate included pus from infected surgical wounds, infected burn wounds, conjunctival swab, aural swab, throat swab, vaginal swab, and urine for microbiological and biochemical analysis of methicillinresistant S. aureus. Results: All the 264 cases of staphylococcal species isolated from different clinical specimens were subjected to coagulase test. It was observed that out of 264 strains of staphylococci isolated from different sites, 165 strains (62.5\%) were coagulase positive and 99 strains (37.5\%) were coagulase negative by tube method. Out of the 165 strains of coagulase-positive staphylococci, maximum isolation was obtained from pus 74 followed by throat swab 55, aural swab 21, vaginal 4, conjunctival swab 9 , and urine 2. All the 165 cases of coagulase-positive Staphylococcus isolated from different clinical specimens were studied for hemolysis, mannitol fermentation, pigment production, and phosphatase production. Out of these 165 strains, 162 (98\%) strains produced $\beta$-hemolysis on blood agar medium. Pigment production was noted in 160 (97\%) of cases. Majority of strains produced characteristic golden yellow pigment on nutrient agar plate. A total of 155 (94\%) strains of staphylococci fermented mannitol with the production of acid only. Phosphatase production was observed in 157 (95\%) strains of pathogenic staphylococci. Conclusion: Considering the above-mentioned pathogenicity test, it was observed that coagulase test was the single most reliable test, though coagulase-negative staphylococci are sometimes pathogenic too.
\end{abstract}

Keywords: Methicillin-resistant Staphylococcus aureus, microbiological and biochemical analysis, Staphylococci

Asian Pac. J. Health Sci., (2020); DOI: 10.21276/apjhs.2020.7.3.10

\section{INTRODUCTION}

Staphylococci are Gram-positive cocci belonging to the family Micrococcaceae of which Staphylococcus aureus is the only coagulase-positive species capable of causing infection in humans. It is one of the major causes of hospital cross infection. In the 1930s, sulfonamides were used in the treatment of staphylococcal infection, but they failed to gain popularity because of their decreased activity in the presence of pus and rapid acquisition of antibiotic resistance by the bacteria. ${ }^{[1,2]}$

Methicillin-resistant S. aureus has emerged in the past decade as one of the most important nosocomial pathogens. Infected and colonized patients provide the primary reservoir and transmission is mainly through hospital staff. Risk factors which contribute to methicillin-resistant $S$. aureus are excessive antibiotic usage, prolonged hospitalization, intravascular catheterization, and hospitalization in intensive care unit. Life-threatening sepsis, endocarditis, and osteomyelitis caused by methicillin-resistant S. aureus have been reported..$^{[3,4]}$

Since methicillin-resistant $S$. aureus strains are resistant to multiple antibiotics, there is a possibility of extensive outbreaks which may be difficult to control. Early detection of methicillinresistant Staphylococcus is important from patients and hospitals point of view. Hence, knowledge of methicillin-resistant S. aureus strain and their microbiological and biochemical identification is necessary in selection of appropriate treatment for methicillinresistant S. aureus infection.
'Department of Microbiology, Hazaribagh Medical College, Hazaribagh, Jharkhand, India

${ }^{2}$ Department of General Surgery, ICARE Institute of Medical Sciences and Research, Haldia, West Bengal, India

${ }^{3}$ Department of Microbiology, Rajendra Institute of Medical Sciences, Ranchi, Jharkhand, India

${ }^{4}$ Department of Obstetrics and Gynaecology, Hazaribagh Medical College, Hazaribagh, Jharkhand, India

${ }^{5}$ Department of Anesthesiology, ICARE Institute of Medical Sciences and Research, Haldia, West Bengal, India

Corresponding Author: Dr. Krishna Chander Birua, Department of General Surgery, ICARE Institute of Medical Sciences and Research, Banbishnupur, Purba Medinipur, Haldia, West Bengal - 721 645, India. E-mail: krishnabirua@gmail.com

How to cite this article: Boipai M, Birua KC, Sahu NP, Pandey LB, Beck R, Dinkar S. Microbiological and Biochemical Analysis of Methicillinresistant Staphylococcus aureus Isolated from Patients Admitted in RIMS, Ranchi. Asian Pac. J. Health Sci., 2020; 7(3):39-43

Source of support: Nil

Conflicts of interest: None

Received: 15/12/2019 Revised: 20/01/2020 Accepted: 10/02/2020

\section{Materials and Methods}

The present study was carried out in the Department of Microbiology, Rajendra Institute of Medical Sciences, Ranchi, 
clinical isolates of methicillin-resistant $S$. aureus strains were obtained from admitted patients of Rajendra Institute of Medical Sciences, Ranchi. The sources of isolate included pus from infected surgical wounds, infected burn wounds, conjunctival swab, aural swab, throat swab, vaginal swab, and urine. The patients were at first explained the object of the study and the method of obtaining the specimen so that their full cooperation could be obtained and written informed consent was taken.

\section{Collection of Specimen}

Pus, conjunctival, aural throat, and vaginal swab were collected by means of sterile cotton swab sticks. The sterile cotton swab sticks were moistened with normal saline and rubbed over the infected area taking care not to touch anything outside so as to prevent contamination. Swabs were then aseptically replaced in sterilized test tubes to avoid drying of the material. Efforts were made to inoculate the specimen within $2 \mathrm{~h}$ of collection. Primary inoculation was done on blood agar. The plates after inoculation were incubated at $37^{\circ} \mathrm{C}$ for $24 \mathrm{~h}$. Midstream samples of urine were received in a sterilized vial and inoculated on MacConkey agar. The plates were incubated at $37^{\circ} \mathrm{C}$ for $24 \mathrm{~h}$.

On the next day, colonies of staphylococci were identified on blood agar plate by its characteristics appearance. The colonies of staphylococci were of small size, opaque, and surrounded by a zone of $\beta$-hemolysis. Colonies of staphylococci on MacConkey agar were smaller in size, pink in color due to lactose fermentation. In general, mixed cultures were obtained from samples collected from different infected sites. To get pure Staphylococcus species, suspected colony from the mixed culture was subcultured onto the nutrient agar and incubated at $37^{\circ} \mathrm{C}$ for $24 \mathrm{~h}$.

The colonies of Staphylococcus on nutrient agar were $2-3 \mathrm{~mm}$ in diameter, with a smooth glistening surface, entire edge, butyrous in consistency, opaque, and pigmented appearance. Most of them were golden yellow in color but some of them were white and lemon yellow in color.

Smears were prepared from discrete colonies on nutrient agar with normal saline. The air-dried and heat fixed smears were stained by Gram staining method and examined microscopically. Gram-positive cocci arranged in grape-like clusters were identified as Staphylococcus.

Isolated colony was picked up from an 18 to $24 \mathrm{~h}$ culture on nutrient agar with the help of a sterile platinum wire loop and placed on a clean glass slide. A drop of $3 \%$ hydrogen peroxide was added over the colony on the slide. Immediate bubbling was recorded as a positive result. This test differentiates between the catalase-positive Micrococcaceae species are catalase positive.

The organisms were inoculated on blood agar plate, incubated at $37^{\circ} \mathrm{C}$ for $24 \mathrm{~h}$. Then, it was looked for hemolysis. Usually, there was beta $(\beta)$ type of hemolysis, that is, a clear zone of hemolysis around the colonies.

On nutrient agar plate, the organism was inoculated and incubated overnight, thus colonies obtained were observed for the color of pigment produced by staphylococci. Usually, golden yellow pigment was produced by pathogenic strains of staphylococci. Pigment production was enhanced when $1 \%$ glycerol monoacetate or mild was incorporated in the medium or when the temperature was kept at $22^{\circ} \mathrm{C}$.

Organisms were inoculated in Mannitol salt agar containing nutrient agar with $1 \%$ mannitol and $7.5 \%$ sodium chloride and incubated at $37^{\circ} \mathrm{C}$ for 24 h. S. aureus produces yellowish colonies on the medium due to fermentation of mannitol with the production of acid. Other staphylococcal species produces pink colonies.

Phenolphthalein-diphosphate was added to nutrient agar plate and organisms were inoculated in this plate and allowed to grow by incubating at $37^{\circ} \mathrm{C}$ for $24 \mathrm{~h}$. Then, $0.1 \mathrm{ml}$ of ammonia solution was placed in the lid of Petri dish and the dish with culture was placed over it for a minute. The pathogenic strains of staphylococci produce the enzyme phosphatase which liberate free phenolphthalein from phenolphthalein-diphosphate which were detected by exposing the growth with ammonia vapor, turning the growth bright pink, indicating a positive test.

\section{Results}

All the 264 cases of staphylococcal species isolated from different clinical specimens were subjected to coagulase test [Table 1 and Figure 1]. It was observed that out of 264 strains of staphylococci isolated from different sites, 165 strains (62.5\%) were coagulase

Table 1: Number of isolation of Staphylococcus sp. from different clinical specimens

\begin{tabular}{lc}
\hline Specimens & Number \\
\hline Pus and wound & 84 \\
Throat swab & 78 \\
Aural swab & 39 \\
Conjunctival swab & 32 \\
Urine & 18 \\
Vaginal swab & 13 \\
\hline
\end{tabular}

Table 2: Results of coagulase test of 264 strains of staphylococci isolated from different clinical specimens

\begin{tabular}{lcc}
\hline Specimens & Coagulase +ve staph. & Coagulase -ve staph. \\
\hline Pus and wound & 74 & 10 \\
Throat swab & 55 & 23 \\
Aural swab & 21 & 18 \\
Conjunctival swab & 9 & 23 \\
Urine & 2 & 16 \\
Vaginal swab & 4 & 9 \\
\hline
\end{tabular}

Table 3: Results of hemolysis on blood agar, mannitol fermentation, pigment production, and phosphatase production of 165 strains of coagulase-positive Staphylococcus

\begin{tabular}{lcc}
\hline Specimens & Positive strains & Negative strains \\
\hline Hemolysis & 162 & 3 \\
Mannitol fermentation & 155 & 10 \\
Pigment production & 160 & 5 \\
Phosphatase production & 157 & 8 \\
\hline
\end{tabular}

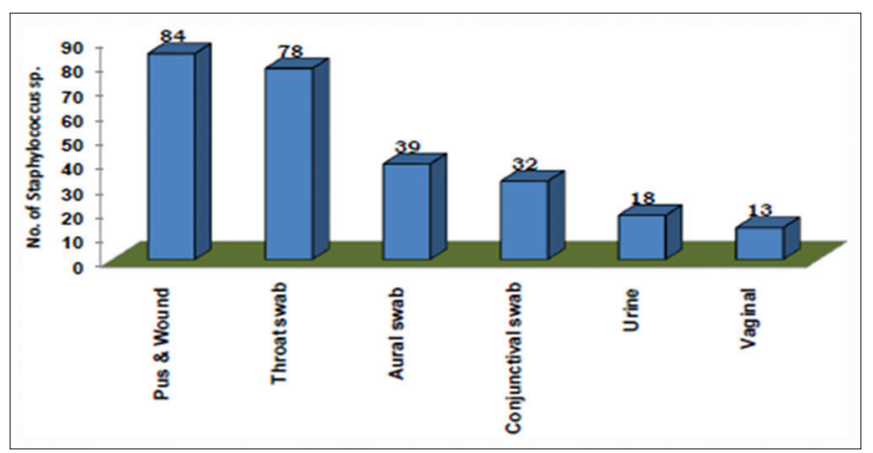

Figure 1: Number of isolation of Staphylococcus sp. from different clinical specimens 


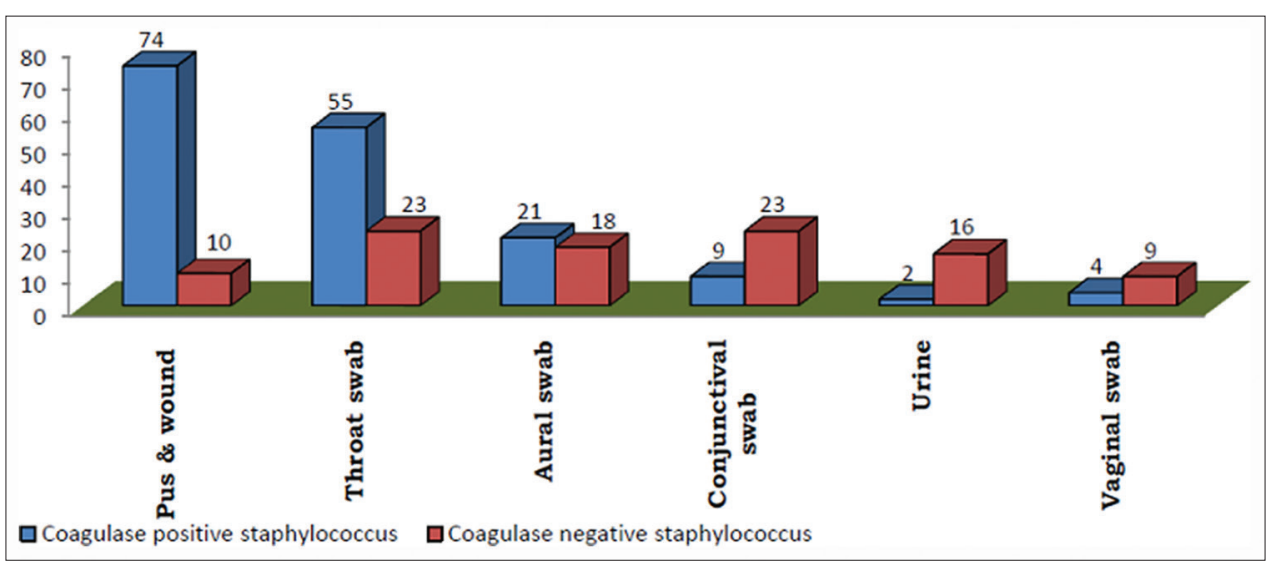

Figure 2: Results of coagulase test of 264 strains of staphylococci isolated from different clinical specimens

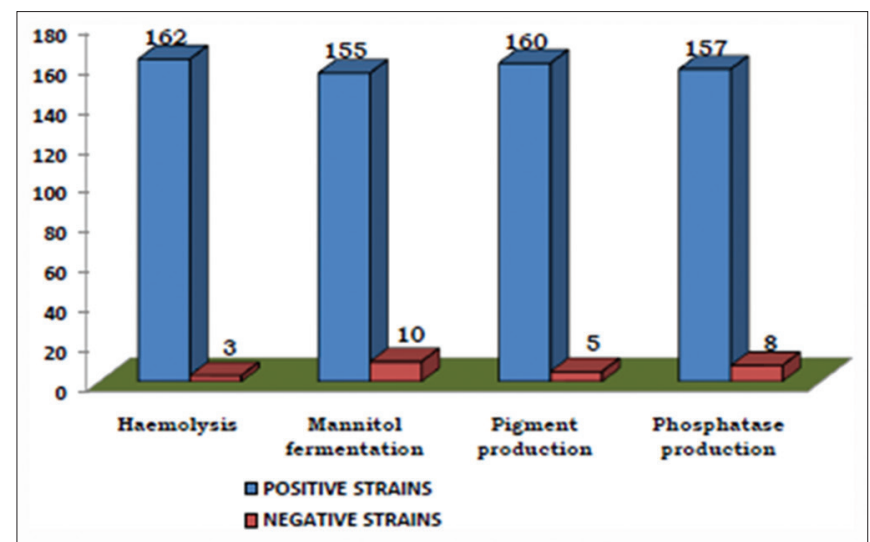

Figure 3: Results of hemolysis on blood agar, mannitol fermentation, pigment production, and phosphatase production of 165 strains of coagulase-positive Staphylococcus

positive and 99 strains (37.5\%) were coagulase negative by tube method. Out of the 165 strains of coagulase-positive staphylococci, maximum isolation was obtained from pus 74 followed by throat swab 55, aural swab 21, vaginal 4, conjunctival swab 9, and urine 2 [Table 2 and Figure 2].

All the 165 cases of coagulase-positive Staphylococcus isolated from different clinical specimens were studied for hemolysis, mannitol fermentation, pigment production, and phosphatase production. Out of these 165 strains, 162 (98\%) strains produced $\beta$-hemolysis on blood agar medium. Pigment production was noted in $160(97 \%)$ of cases. Majority of strains produced characteristic golden yellow pigment on nutrient agar plate. A total of 155 (94\%) strains of staphylococci fermented mannitol with the production of acid only. Phosphatase production was observed in 157 (95\%) strains of pathogenic staphylococci [Table 3 and Figure 3].

\section{Discussion}

The present work is "study of methicillin resistance S. aureus isolated from patient admitted in RIMS and testing their sensitivity to antimicrobial drugs." Samples were collected from different sources such as pus, throat, ear, conjunctiva, vagina, and urine. Various criteria have been proposed to differentiate pathogenic from non-pathogenic strains of staphylococcus. However, coagulase and phosphatase production, ability to hemolyse red blood cell, fermentation of mannitol, and pigment production have been commonly used..$^{[5,6]}$

The pathogenic strains of staphylococcus were studied for their resistance to methicillin on Mueller-Hinton agar supplemented with $5 \%$ sodium chloride using oxacillin or methicillin disc. Recent sensitivity pattern of methicillin resistance $S$. aureus was studied against the available newer antibiotics. Hence, knowledge of the methicillin-resistant $S$. aureus strains and their sensitivity pattern will help in proper treatment of such patients.

Identification of pathogenicity was of prime importance because of the fact that staphylococci are ubiquitous and normal inhabitant and multiply in the anterior nares, throat, and skin of healthy people in addition to their presence in pyogenic lesion. It is also noted that many strains are relatively harmless in comparison to those isolated from infective lesions. ${ }^{[7]}$

In the present study, 264 strains of staphylococci isolated from different clinical samples were subjected to coagulase test, out of which 165 strains (62.5\%) were coagulase-positive staphylococci. The study of coagulase-positive staphylococci is being compared here.

\begin{tabular}{lcc}
\hline Workers & Year & $\begin{array}{c}\text { Coagulase-positive } \\
\text { staphylococci (\%) }\end{array}$ \\
\hline Raymond and Traub et al. ${ }^{[8]}$ & 1970 & 83.20 \\
Deepak et al. ${ }^{\left[{ }^{\prime}\right]}$ & 1999 & 80.3 \\
Majumdar al ${ }^{[10]}$ & 2001 & 71.2 \\
Rajput et al. ${ }^{[11]}$ & 2008 & 60.35 \\
Present study & 2009 & 62.5 \\
\hline
\end{tabular}

From the above observation, it is apparent that in the present study, 165 strains (62.5\%) produced coagulase enzyme and remaining 99 strains $(37.5 \%)$ were coagulase negative by tube method, 160 strains (60.6\%) were coagulase positive by slide method. This figure correlated will with the positive staphylococci and $39.64 \%$ coagulase-negative staphylococci were observed.

The study of coagulase-positive S. aureus in different clinical samples is being compared.

\begin{tabular}{lccc}
\hline Year & 1999 & 2008 & 2009 \\
\hline Specimen & Deepak & Anuradha & Present \\
& et al..$^{[9]}$ & et al. $^{[11]}$ & study \\
Pus & $88.19 \%$ & $72 \%$ & $88 \%$ \\
Throat swab & $70.5 \%$ & $73.12 \%$ & $70 \%$ \\
Aural swab & $78 \%$ & $55.56 \%$ & $54 \%$ \\
Conjunctival swab & $33 \%$ & $27.27 \%$ & $28 \%$ \\
Urine & $12.5 \%$ & $15.6 \%$ & $11 \%$ \\
Vaginal swab & $33 \%$ & $28.57 \%$ & $31 \%$ \\
\hline
\end{tabular}


In the present study, the rate of occurrence of $S$. aureus in pus was $88 \%$, in urine $(11 \%)$, and in vaginal swab $(31 \%)$; this figure correlated will with the study of pathogenic staphylococci by Deepak et al. ${ }^{[9]}$ showing rate of occurrence of $S$. aureus in pus $(88.19 \%)$, in urine $(12.5 \%)$, and in vaginal swab (33\%).

In the present study, the rate of occurrence of $S$. aureus in throat swab was $70 \%$, in conjunctival swab (28\%), and in aural swab $(54 \%)$, this figure correlated with the study of pathogenic staphylococci $(2008)^{[11]}$ showing rate of occurrence of S. aureus in throat swab $(73.12 \%)$, in conjunctival swab $(27.27 \%)$, and in aural swab (55.56\%). Among the procedures available to detect coagulase-positive staphylococci, slide coagulase test advocated by William and Harper ${ }^{[12]}$ is very simple, economical, and easy to perform in comparison to tube coagulase test. ${ }^{[13]}$

Although simple and in general useful, the slide coagulase test should not be used as a sole technique in the diagnostic laboratory. False-positive results are caused by citrate utilizing bacteria such as enterococci and pseudomonas. Therefore, in doubtful cases, the tube coagulase test should be done and those strains which appear to be pathogenic staphylococci and fails to give slide test positive should be subjected to tube method. ${ }^{[14]}$ Hence, it was concluded that coagulase production should be the single test to distinguish pathogenic strains of staphylococci. However, nowadays, coagulase-negative strains are also found to be responsible for disease production. Breckinridge and Bergdoll described an outbreak of acute gastroenteritis in which responsible organism was enterotoxin producing coagulasenegative staphylococcus. ${ }^{[15]}$

\section{Mannitol Fermentation}

It has been used by many workers to distinguish the pathogenic strains of staphylococci from non-pathogenic one. This test has long been considered an important test in the classification of pathogenic staphylococci. In the present work out of 165 strains of coagulase-positive staphylococci studied, 155 strains (94\%) were mannitol fermenter producing acid only and 10 strains (6\%) were non-fermenter of mannitol. This figure correlated well to the study of Raymond and Traub ${ }^{[8]}$ who observed $83.20 \%$ coagulase-positive mannitol fermenter. Mannitol fermentation is now not considered an important test to differentiate non-pathogenic staphylococci from pathogenic staphylococci because good percentage coagulasenegative staphylococci were found as mannitol fermenter.

\section{Pigment Production}

Isolated strain of staphylococci was also observed for pigment production. The study of pigment production by coagulasepositive staphylococci is being compared hereby.

\begin{tabular}{lcc}
\hline Workers & Year & Pigment-producing staphylococci (\%) \\
\hline Goyle et al. ${ }^{[16]}$ & 2002 & 83 \\
Anuradha et al. ${ }^{[11]}$ & 2004 & 93.33 \\
Present study & 2009 & 97 \\
\hline
\end{tabular}

In the present study out 165 strains of coagulase-positive staphylococci studied, 160 (97\%) strains of staphylococci produced golden yellow and yellow pigment, 5 strains (3\%) did not produce pigment. The present study correlates well with the study of pathogenic staphylococci by Anuradha et al. ${ }^{[11]}$ who observed $93.33 \%$ of coagulase-positive staphylococci producing golden yellow and yellow pigment and rest $6.67 \%$ strains did not produce pigment.
However, pigment production is dependent on many factors. Oxygen is important for pigment production; under anaerobic condition, the growth is colorless. An agar medium containing $33 \%$ milk is recommended for its study. The pigment production is optimal at 22 degree $\mathrm{C}$ than at $37^{\circ} \mathrm{C}$. Thus, it is obvious that optimum temperature for pigment production does not coincide with optimum temperature for growth. ${ }^{[12]}$

\section{$\beta$-Hemolysis on Blood Agar}

The study of $\beta$-hemolysis on blood agar by coagulase-positive staphylococci is being compared hereby.

\begin{tabular}{lcc}
\hline Workers & Year & B-Hemolysis on blood agar (\%) \\
\hline Christie and Keogh $^{[17]}$ & 1940 & 90 \\
Anuradha et al. ${ }^{[11]}$ & 2004 & 96.67 \\
Present study & 2009 & 98 \\
\hline
\end{tabular}

In the present study, out of 165 strains of staphylococci studied, 162 (98\%) strains of staphylococci produced $\beta$-hemolysis on sheep blood agar medium and remaining $3(2 \%)$ strains of staphylococci did not produce clear-cut hemolysis on blood agar plate. The above result correlated well with the study of pathogenic staphylococci by Anuradha et al. who observed $96.67 \%$ of coagulase-positive staphylococci producing $\beta$-hemolysis on blood agar medium. The above result also correlates with a study of Christie and Keogh's ${ }^{[17]}$ who observed that $90 \%$ of pathogenic strains produced $\beta$-hemolysis on blood agar.

Abramson and Friedman ${ }^{[18]}$ observed on 60 coagulase in hospital environment and noted that $50 \%$ of these strains produced hemolysis on sheep blood agar plate. This observation gives clear-cut indication that there is intimate relationship between pathogenicity of staphylococci and hemolysis of sheep RBC. Alpha-toxin has been playing a great role in producing lesions of pathogenic staphylococci. Its main significance in pathogenicity is that of producing tissue damage after the establishment of a focus infection. Schwabacher et al. ${ }^{[19]}$ reported that $92.1 \%$ coagulase-positive staphylococci produced alpha-toxin. Thus, it was suggested that alpha-lysin production is confined to pathogenic staphylococci which is conveniently demonstrable with rabbit's red blood corpuscles. Barber ${ }^{[20]}$ concluded that alpha-toxin, fibrinolysin, and pigment production probably all play an important part, it is difficult to single out any one of them as essential for pathogenicity and quantitative estimation of any one these cannot give much indication of virulence of the organism. There is evidence to suggest that broad spectrum of toxin production is of more significance than high production of any one. ${ }^{[20]}$

\section{Phosphatase Production}

The study of phosphatase production by pathogenic staphylococci is being compared hereby.

\begin{tabular}{lcc}
\hline Workers & Year & $\begin{array}{c}\text { Phosphatase production by } \\
\text { pathogenic staphylococci (\%) }\end{array}$ \\
\hline Barber and Kuper & $121]$ & 53.15 \\
Anuradha et al. ${ }^{[1]]}$ & 2051 & 83.5 \\
Present study & 2004 & 95 \\
\hline
\end{tabular}

In the present study, out of 165 strains of staphylococci studied, 157 (95\%) strains of pathogenic staphylococci produced phosphatase in the phenolphthalein agar medium and rest 8 (5\%) strains did not produce phosphatase. The present study correlates 
well with the study of pathogenic staphylococci showing $83.5 \%$ strains producing phosphatase followed by the study of Barber and Kuper ${ }^{[21]}$ showing $53.15 \%$ of S. aureus producing phosphatase.

Gelatin liquefaction test, egg yolk reaction, and serological typing could not be performed due to certain difficulties. However, it has been reported in literature that these tests have no advantage over coagulase test. Similarly, animal pathogenicity test is also not satisfactory and not of much help because human being and laboratory animals react differently to staphylococcal infection. ${ }^{[20]}$ Hence, bacteriology is in favor of accepting coagulase test as the most important indicator of distinguishing pathogenic staphylococci from non-pathogenic strains.

Staphylococcus occurs on the body surface of many species of mammals and appears to be their normal habitat. S. aureus is carried in the nasopharynx of some $30 \%$ of persons, many of whom are also skin carriers. Majority of staphylococci live harmlessly on the surface of body, some penetrate the skin and cause local damage, and few may invade blood stream and give rise to serious manifestation, especially when resistance of the host is impaired. Skin is an excellent barrier to bacterial infection. Invasion of skin and subcutaneous tissue by staphylococci occur most commonly when the continuity of the epithelium is disrupted. Thus, abrasions, wounds, burns, etc., are particularly prone to become infected by staphylococci. ${ }^{[22]}$ When parenteral injection is performed without attention to aseptic techniques, serious local staphylococcal infection not uncommonly followed by bacteremia and often metastatic abscess occurs.

\section{ConcLusion}

A total of 500 samples were collected from patients admitted in RIMS out of which in 264 cases (52.8\%), staphylococcal species were isolated. All the staphylococcal strains were studied for their morphological and cultural characters, pigment production, betahemolysis, mannitol fermentation, coagulase, and phosphatase production.

All the 264 cases of staphylococcal species isolated from different clinical specimens were subjected to coagulase test. It was observed that out of 264 strains of staphylococci isolated from different sites, 165 strains (62.5\%) were coagulase positive and 99 strains (37.5\%) were coagulase negative by tube method. Out of the 165 strains of coagulase-positive staphylococci, maximum isolation was obtained from pus 74 followed by throat swab 55, aural swab 21, vaginal 4, conjunctival swab 9, and urine 2.

All the 165 cases of coagulase-positive Staphylococcus isolated from different clinical specimens were studied for hemolysis, mannitol fermentation, pigment production, and phosphatase production. Out of these 165 strains, 162 (98\%) strains produced $\beta$-hemolysis on blood agar medium. Pigment production was noted in 160 (97\%) of cases. Majority of strains produced characteristic golden yellow pigment on nutrient agar plate. A total of 155 (94\%) strains of staphylococci fermented mannitol with the production of acid only. Phosphatase production was observed in 157 (95\%) strains of pathogenic staphylococci.

Considering the above-mentioned pathogenicity test, it was observed that coagulase test was the single most reliable test, though coagulase-negative staphylococci are sometimes pathogenic too.

\section{References}

1. Abramson C. Staphylococcal enzymes. In: Cohen JO, editor. The Staphylococci. New York: John Wiley; 1972. p. 187-248.

2. Taylor TA, Unakal CG. Staphylococcus aureus. Treasure Island, FL: StatPearls Publishing; 2020.

3. Lakhundi S, Zhang K. Methicillin-resistant Staphylococcus aureus: Molecular characterization, evolution, and epidemiology. Clin Microbiol Rev 2018;31:e00020-18.

4. Siddiqui $\mathrm{AH}$, Koirala J. Methicillin Resistant Staphylococcus aureus (MRSA) Treasure Island, FL: StatPearls Publishing; 2020.

5. Karmakar A, Dua P, Ghosh C. Biochemical and molecular analysis of Staphylococcus aureus clinical isolates from hospitalized patients. Can J Infect Dis Med Microbiol 2016;2016:9041636.

6. Adhikari R, Pant ND, Neupane S, Neupane M, Bhattarai R, Bhatta S, et al. Detection of methicillin resistant Staphylococcus aureus and determination of minimum inhibitory concentration of vancomycin for Staphylococcus aureus isolated from pus/wound swab samples of the patients attending a tertiary care Hospital in Kathmandu, Nepal. Can J Infect Dis Med Microbiol 2017;2017:2191532.

7. Murray PR, Baron EJ, Jorgensen JH, Pfaller MA, Yolken RH. Manual of Clinical Microbiology. $8^{\text {th }}$ ed. Washington, DC, USA: American Society for Microbiology; 2003.

8. Raymond EA, Traub WH. Identification of staphylococci isolated from clinical material. Appl Microbiol 1970;19:919-22.

9. Deepak S, Samant SA, Urhekar AD. Study of coagulase positive and negative staphylococci in clinical samples. Indian J Med Sci 1999;53:425-8.

10. Majumder D, Bordoloi JN, Phukan AC, Mahanta J. Antimicrobial susceptibility pattern among methicillin resistant Staphylococcus isolates in Assam. Indian J Med Microbiol 2001;19:138-40.

11. Rajput A, Singh KP, Kumar V, Sexena R, Singh RK. Antibacterial resistance pattern of aerobic bacteria isolates from burn patients in tertiary care hospital. Biomed Res 2008;19:1-4.

12. Williams RE, Harper GJ. Determination of coagulase and alphahaemolysin production by staphylococci. Br J Exp Pathol 1946;27:72-81.

13. Gillespie EH. The routine use of coagulase test for staphylococci. Mon Bull Emerg Public Health Lab Serv 1943;2:19.

14. Kateete DP, Kimani CN, Katabazi FA, Okeng A, Okee MS, Nanteza A et al. Identification of Staphylococcus aureus: DNase and mannitol salt agar improve the efficiency of the tube coagulase test. Ann Clin Microbiol Antimicrob 2010;9:23.

15. Breckinridge JC, Bergdoll MS. Outbreak of food-borne gastroenteritis due to a coagulase-negative enterotoxin-producing staphylococcus. N Engl J Med 1971;284:541-3.

16. Goyal R, Das S, Mathur M. Colonisation of methicillin resistant Staphylococcus aureus among health care workers in a tertiary care hospital of Delhi. Indian J Med Sci 2002;56:321-4.

17. Christie R, Keogh EV. Physiological and serological characteristics of staphylococci of human origin. J Pathol Bacteriol 1940;51:189-97.

18. Abramson C, Friedman H. Enzymatic activity of primary isolates of staphylococci in relation to antibiotic resistance and phage type. J Infect Dis 1967;117:242-8.

19. Schwabacher H, Cunliffe AC, Willmams RE, Harper GJ. Hyaluronidase production by staphylococci. Br J Exp Pathol 1945;26:124-9.

20. Barber M. Methicillin-resistant staphylococci. J Clin Pathol 1961;14:385-93.

21. Barber F, Kuper SW. Identification of Staphylococcus pyogenes by the phosphatase reaction. J Pathol Bacteriol 1951;63:65-8.

22. Cluff LE, Reynolds RC, Page DL, Breckenridge JL. Staphylococcal bacteremia and altered host resistance. Ann Intern Med 1968;69:859-73. 